\title{
Nontrivial solutions for a quasilinear elliptic system
}

\author{
Xiyou Cheng* and Lu Yang
}

${ }^{\text {*Correspondence: }}$ chengxy@|zu.edu.cn School of Mathematics and Statistics, Lanzhou University, Lanzhou, 730000, P.R. China

\begin{abstract}
In this paper, we deal with the existence of three nontrivial solutions for the potential system of $p$-Laplacian equations with homogeneous Dirichlet boundary conditions. Applying the Nehari procedure and abstract linking theorem on a product space, we give a linking structure for our variational problem, and then, combining with the classical minimax principle, we obtain three nontrivial critical values for the relevant energy functional.
\end{abstract}

MSC: $35 J 20 ; 35 J 25$

Keywords: elliptic equations; Nehari manifold; linking theorem; minimax principle

\section{Introduction}

In this paper, we are concerned with the multiplicity of nontrivial solutions for the following system of quasilinear equations:

$$
\begin{cases}-\Delta_{p_{1}} u=f_{1}(x, u)+\lambda h_{1}(x, u, v), & \text { in } \Omega, \\ -\Delta_{p_{2}} v=f_{2}(x, v)+\lambda h_{2}(x, u, v), & \text { in } \Omega, \\ u=v=0, & \text { on } \partial \Omega,\end{cases}
$$

where $-\Delta_{p_{i}} u=\operatorname{div}\left(|\nabla u|^{p_{i}-2} \nabla u\right)\left(p_{i}>1\right), \Omega$ is a bounded smooth domain in $\mathbb{R}^{N}(N \geq 3)$, $f_{i}: \Omega \times \mathbb{R}^{+} \rightarrow \mathbb{R}^{+}, h_{i}: \Omega \times \mathbb{R}^{+} \times \mathbb{R}^{+} \rightarrow \mathbb{R}^{+}$are Carathéodory functions $(i=1,2)$, and there exists a function $H \in C^{1}(\Omega \times \mathbb{R} \times \mathbb{R}, \mathbb{R})$ such that

$$
\nabla H(x, u, v)=\left(H_{u}(x, u, v), H_{v}(x, u, v)\right)=\left(h_{1}(x, u, v), h_{2}(x, u, v)\right) .
$$

In recent years, many authors have studied the existence of nontrivial solutions for Laplacian systems and $p$-Laplacian systems, see [1-5] and the references therein. Usually the authors change the problem into the critical point problem of the corresponding energy functional and then apply the critical point theory or the variational method, or they change it into the fixed point problem of the corresponding compactly continuous mapping and then apply topological degree theory or the method of lower and upper solutions. For instance, in [2] Costa and Magalhaes unified the cooperative and noncooperative Laplacian systems, and they got the existence of nontrivial solutions via the variational approach; in [1] Conti et al. dealt with the competitive Laplacian system, and they established the existence of positive solutions by the Nehari procedure, critical point theory, and topological degree theory; in $[3,4]$ the authors studied the sublinear $p$-Laplacian systems, and

○2014 Cheng and Yang; licensee Springer. This is an Open Access article distributed under the terms of the Creative Commons Attribution License (http://creativecommons.org/licenses/by/2.0), which permits unrestricted use, distribution, and reproduction in any medium, provided the original work is properly cited. 
they obtained the existence of positive solutions by the method of lower and upper solutions and Leray-Schauder degree theory, respectively; in [5] Zhang and Zhang considered the existence of nontrivial solutions for nonlinear Laplacian systems and $p$-Laplacian systems applying the direct variational method. More recently, some authors discussed the multiplicity of nontrivial solutions for Laplacian systems and $p$-Laplacian systems, see [6$12]$ and references therein. In $[6,11,12]$, the authors provided the existence results of three nontrivial solutions for system (1.1) with one parameter for the case $p=2$, where the main methods used are the Nehari procedure and the linking theorem on product space. In [8] Motreanu and Zhang consider a general noncoercive quasilinear elliptic system, they establish the existence of two opposite constant sign solutions; in the case where the system has a variational structure, under the proper hypotheses, they obtain a third nontrivial solution, which is sign changing in the sense that one cannot have both components of the new solution of the same constant sign; their approach relies on a suitable method of sub-supersolutions combined with truncation and variational arguments that do not require a subcritical growth condition. In [9] Shen and Zhang established the existence of two positive solutions for multi-parameter $p$-Laplacian systems with critical exponents by use of the Nehari procedure and the variational approach. In $[7,10]$, the present author and coauthors dealt with a class of Laplacian systems with superlinear and sublinear terms applying the fixed point index formula on a product cone, and they obtained the existence and multiplicity of positive solutions.

Motivated by some ideas in $[1,7,9,12]$, we shall deal with the existence of nonnegative solutions (especially, positive solutions) for system (1.1) with superlinear and subcritical nonlinear terms. It is well known that the Ambrosetti-Rabinowitz type result (see [13, 14]) can be extended to system (1.1) with superlinear and subcritical nonlinear terms by imposing the Ambrosetti-Rabinowitz conditions and other proper conditions on nonlinear terms. Now, we have a natural question of when system (1.1) has multiple nonnegative solutions. In order to obtain the multiplicity of nonnegative solutions for system (1.1), we need only to construct the multiple critical values of the corresponding energy functional. For this matter, first we get two ground states in view of the Nehari procedure, establish a linking structure by the abstract linking theorem on the product space, and then construct the third critical value by the classical minimax principle.

The present paper is organized as following. In Section 2, we provide a linking structure for our variational problem (see Theorem 2.2); in Section 3, we verify that the energy functional satisfies the (P.S.) condition (see Theorem 3.1); in Section 4, we prove the existence of three nonnegative solutions for system (1.1) as $\lambda$ is small enough, and, in addition, one of them is positive if the equations excluding coupled terms have both a unique positive solution (see Theorem 4.1).

\section{Linking structure}

In this section, we list some preliminaries, including the abstract linking theorem on the product space and the concept of Nehari manifold, and then we give a linking structure, which is useful for constructing the critical value of the functional associated with system (1.1).

Definition 2.1 [12] Let $f$ be a real functional on Banach space $X$ and $c$ be a real constant, we say that $S_{f}^{c}=\{u \in X \mid f(u)=c, u \neq 0\}$ has the sphere property, if the following hypotheses are satisfied: 
$\left(\mathrm{S}_{1}\right) f$ is continuous on $X$;

$\left(\mathrm{S}_{2}\right)$ there is a homeomorphic mapping between $S_{f}^{c}$ and unit sphere of $X$;

$\left(\mathrm{S}_{3}\right)$ for any fixed $u \in X \backslash\{0\}$, the equation $f(t u)=c$ has a unique solution $t \in(0, \infty)$;

$\left(\mathrm{S}_{4}\right) X$ is separated into two open connected subsets by $S_{f}^{c}$ and the origin is contained in one of the subsets.

Theorem 2.1 [12] Let X, Y be Banach spaces with the following direct sum decomposition:

$$
X=X_{1} \oplus X_{2}, \quad Y=Y_{1} \oplus Y_{2},
$$

where $X_{1}, Y_{1}$ are finite dimensional subspaces of $X, Y$, respectively. Let $f, g$ be the real functionals on $X, Y$, respectively, $c, d$ be two real constants and $S_{f}^{c}, S_{g}^{d}$ have the sphere property. Take $\left(e_{x}, e_{y}\right) \in X_{2} \times Y_{2}$ such that $f\left(e_{x}\right)>c$ and $g\left(e_{y}\right)>d$. Denote

$$
\begin{aligned}
& Q^{X}=\left\{u+t e_{x} \mid u \in X_{1} \cap B_{R_{1}}, t \in[0,1]\right\}, \quad Q^{Y}=\left\{v+s e_{y} \mid v \in Y_{1} \cap B_{R_{2}}, s \in[0,1]\right\} ; \\
& S_{1}^{X_{2}}=S_{f}^{c} \cap X_{2}, \quad S_{2}^{Y_{2}}=S_{g}^{d} \cap Y_{2} ; \quad Q=Q^{X} \times Q^{Y} \text { and } S=S_{1} \times S_{2},
\end{aligned}
$$

then $\partial Q$ links $S$.

Definition 2.2 [15] Assume that $\varphi \in C^{1}(X, \mathbb{R})$ is such that $\varphi^{\prime}(0)=0$, then the constraint set $N=\left\{u \in X \mid\left\langle\varphi^{\prime}(u), u\right\rangle=0, u \neq 0\right\}$ is called a Nehari manifold of $X$.

Let $F_{i}(x, u)=\int_{0}^{u} f_{i}(x, s) \mathrm{d} s$, and

$$
\varphi_{i}(u)=\frac{1}{p_{i}} \int_{\Omega}|\nabla u|^{p_{i}} \mathrm{~d} x-\int_{\Omega} F_{i}(x, u) \mathrm{d} x, \quad u \in W_{0}^{1, p_{i}}(\Omega), i=1,2 .
$$

Denote by $N_{i}$ the Nehari manifold of $\varphi_{i}$ on $X_{i}=W_{0}^{1, p_{i}}(\Omega)$; we have the following.

Lemma 2.1 Assume that the following conditions are satisfied:

$\left(\mathrm{F}_{1}\right) f_{i} \in C\left(\bar{\Omega} \times \mathbb{R}^{+}, \mathbb{R}^{+}\right)$, and there exist constants $q_{i} \in\left(p_{i}, p_{i}^{*}\right)$ and $C_{i}>0$ such that

$$
\left|f_{i}(x, u)\right| \leq C_{i}\left(|u|^{p_{i}-1}+|u|^{q_{i}-1}\right) \quad \text { for all }(x, u) \in \Omega \times \mathbb{R}^{+} ;
$$

$\left(\mathrm{F}_{2}\right)$ there exist constants $\alpha_{i}>\max \left\{p_{1}, p_{2}\right\}$ such that

$$
0<\alpha_{i} F_{i}(x, u) \leq u f_{i}(x, u) \quad \text { for any } x \in \Omega, u>0
$$

$\left(\mathrm{F}_{3}\right) \lim \sup _{u \rightarrow 0} \frac{f_{i}(x, u)}{|u|^{p_{i}-2} u}<\delta_{1, p_{i}}$ uniformly w.r.t. $x \in \Omega$, here $\delta_{1, p_{i}}$ is the first eigenvalue of the operator $-\Delta_{p_{i}}$ on $W_{0}^{1, p_{i}}(\Omega)$;

$\left(\mathrm{F}_{4}\right) f_{i}(x, u) / u^{p_{i}-1}$ is an increasing function of $u$ on $\mathbb{R}^{+} \backslash\{0\}$.

Then the Nehari manifolds $N_{1}, N_{2}$ have the sphere property.

Proof It is very similar to the proof of Lemma 4.1 on page 72 in [15], so we omit it.

Theorem 2.2 Assume that $f_{1}, f_{2}$ satisfy conditions $\left(\mathrm{F}_{1}\right)-\left(\mathrm{F}_{4}\right)$. Let $S=N_{1} \times N_{2}$ and $Q=\left\{\right.$ te $_{1} \mid$ $t \in[0,1]\} \times\left\{s e_{2} \mid s \in[0,1]\right\}$, here $e_{i} \in W_{0}^{1, p_{i}}(\Omega)$. If $\varphi_{i}\left(e_{i}\right)<0$, then $\partial Q$ links $S$. 
Proof Let $g_{i}(u)=\left\langle-\varphi_{i}^{\prime}(u), u\right\rangle$, then $S_{g_{i}}^{0}=N_{i}$. Let $h_{i}(t)=-\varphi_{i}\left(t e_{i}\right)$, then $h_{i}(0)=0$ and $h_{i}(1)>0$, and by conditions $\left(\mathrm{F}_{1}\right),\left(\mathrm{F}_{3}\right)$ and $\left(\mathrm{F}_{4}\right)$ it is easy to verify that there exists a unique $t_{i}^{0} \in(0,1)$ such that $h_{i}^{\prime}\left(t_{i}^{0}\right)=0$, and $h_{i}^{\prime}(t)<0$ for $t \in\left(0, t_{i}^{0}\right), h_{i}^{\prime}(t)>0$ for $t \in\left(t_{i}^{0},+\infty\right)$. Thus

$$
g_{i}\left(e_{i}\right)=\left\langle-\varphi_{i}^{\prime}\left(e_{i}\right), e_{i}\right\rangle=h_{i}^{\prime}(1)>0 .
$$

From Lemma 2.1 and Theorem 2.1, $\partial Q$ links $S$.

\section{Palais-Smale condition}

It is well known that the nontrivial critical points of the functional

$$
\psi(u, v)=\varphi_{1}(u)+\varphi_{2}(v)-\lambda \int_{\Omega} H(x, u, v) \mathrm{d} x, \quad(u, v) \in W_{0}^{1, p_{1}}(\Omega) \times W_{0}^{1, p_{2}}(\Omega)
$$

correspond to nontrivial solutions of system (1.1). In the following, we show that the functional $\psi$ satisfies the (P.S.) condition.

Theorem 3.1 Assume that $f_{1}, f_{2}$ satisfy conditions $\left(\mathrm{F}_{1}\right)-\left(\mathrm{F}_{2}\right)$, and $h_{1}, h_{2}$ satisfy the conditions:

$\left(\mathrm{H}_{1}\right)$ there exist constants $q \in\left(1, \min \left\{p_{1}^{*}, p_{2}^{*}\right\}\right)$ and $C_{3}>0$ such that

$$
\left|h_{1}(x, u, v)\right|+\left|h_{2}(x, u, v)\right| \leq C_{3}\left(|u|^{q-1}+|v|^{q-1}+1\right),
$$

$\left(\mathrm{H}_{2}\right)$ there exist constants $\beta_{i} \in\left(1, \min \left\{\alpha_{1}, \alpha_{2}\right\}\right), C_{4}>0$ and $R>0$ such that

$$
u h_{1}(x, u, v)+v h_{2}(x, u, v) \leq C_{4}\left(|u|^{\beta_{1}}+|v|^{\beta_{2}}\right) \quad \text { if }|u|+|v| \geq R,
$$

then the functional $\psi$ satisfies (P.S.) condition.

Proof Let $\left\{\left(u_{n}, v_{n}\right)\right\} \subset W_{0}^{1, p_{1}}(\Omega) \times W_{0}^{1, p_{2}}(\Omega)$ be a sequence such that

$$
\left\{\psi\left(u_{n}, v_{n}\right)\right\} \text { is bounded, and } \psi^{\prime}\left(u_{n}, v_{n}\right) \rightarrow 0 \text { as } n \rightarrow \infty,
$$

we need to prove that $\left\{\left(u_{n}, v_{n}\right)\right\}$ has a strongly convergent subsequence.

Claim I. $\left\{\left(u_{n}, v_{n}\right)\right\}$ is bounded in $W_{0}^{1, p_{1}}(\Omega) \times W_{0}^{1, p_{2}}(\Omega)$.

In fact, from (3.1) there exists a constant $C_{5}>0$ such that

$$
\varphi_{1}\left(u_{n}\right)+\varphi_{2}\left(v_{n}\right)-\lambda \int_{\Omega} H\left(x, u_{n}, v_{n}\right) \mathrm{d} x \leq C_{5},
$$

and for $n$ large enough

$$
\left\{\begin{array}{l}
\int_{\Omega}\left|\nabla u_{n}\right|^{p_{1}} \mathrm{~d} x-\int_{\Omega} u_{n} f_{1}\left(x, u_{n}\right) \mathrm{d} x-\lambda \int_{\Omega} u_{n} h_{1}\left(x, u_{n}, v_{n}\right) \mathrm{d} x \\
\quad \geq-\left(\int_{\Omega}\left|\nabla u_{n}\right|^{p_{1}} \mathrm{~d} x\right)^{1 / p_{1}}, \\
\int_{\Omega}\left|\nabla v_{n}\right|^{p_{2}} \mathrm{~d} x-\int_{\Omega} v_{n} f_{2}\left(x, v_{n}\right) \mathrm{d} x-\lambda \int_{\Omega} v_{n} h_{2}\left(x, u_{n}, v_{n}\right) \mathrm{d} x \\
\quad \geq-\left(\int_{\Omega}\left|\nabla v_{n}\right|^{p_{2}} \mathrm{~d} x\right)^{1 / p_{2}} .
\end{array}\right.
$$


Taking $r \in\left(\max \left\{p_{1}, p_{2}\right\}, \min \left\{\alpha_{1}, \alpha_{2}\right\}\right)$, combining with (3.2) and (3.3), we have

$$
\begin{aligned}
& C_{5}+\left(\int_{\Omega}\left|\nabla u_{n}\right|^{p_{1}} \mathrm{~d} x\right)^{1 / p_{1}}+\left(\int_{\Omega}\left|\nabla v_{n}\right|^{p_{2}} \mathrm{~d} x\right)^{1 / p_{2}} \\
& \geq\left(\frac{1}{p_{1}}-\frac{1}{r}\right) \int_{\Omega}\left|\nabla u_{n}\right|^{p_{1}} \mathrm{~d} x+\left(\frac{1}{p_{2}}-\frac{1}{r}\right) \int_{\Omega}\left|\nabla v_{n}\right|^{p_{2}} \mathrm{~d} x \\
& -\int_{\Omega}\left(F_{1}\left(x, u_{n}\right)-\frac{1}{r} u_{n} f_{1}\left(x, u_{n}\right)\right) \mathrm{d} x-\int_{\Omega}\left(F_{2}\left(x, v_{n}\right)-\frac{1}{r} v_{n} f_{2}\left(x, v_{n}\right)\right) \mathrm{d} x \\
& +\lambda\left[\int_{\Omega} \frac{1}{r}\left(u_{n} h_{1}\left(x, u_{n}, v_{n}\right)+v_{n} h_{2}\left(x, u_{n}, v_{n}\right)\right) \mathrm{d} x-\int_{\Omega} H\left(x, u_{n}, v_{n}\right) \mathrm{d} x\right] \\
& \geq\left(\frac{1}{p_{1}}-\frac{1}{r}\right) \int_{\Omega}\left|\nabla u_{n}\right|^{p_{1}} \mathrm{~d} x+\left(\frac{1}{p_{2}}-\frac{1}{r}\right) \int_{\Omega}\left|\nabla v_{n}\right|^{p_{2}} \mathrm{~d} x \\
& +\frac{\alpha_{1}-r}{r} \int_{\Omega} F_{1}\left(x, u_{n}\right) \mathrm{d} x+\frac{\alpha_{2}-r}{r} \int_{\Omega} F_{2}\left(x, v_{n}\right) \mathrm{d} x \\
& +\lambda\left[\int_{\Omega} \frac{1}{r}\left(u_{n} h_{1}\left(x, u_{n}, v_{n}\right)+v_{n} h_{2}\left(x, u_{n}, v_{n}\right)\right) \mathrm{d} x-\int_{\Omega} H\left(x, u_{n}, v_{n}\right) \mathrm{d} x\right]-C_{6} \\
& \geq\left(\frac{1}{p_{1}}-\frac{1}{r}\right) \int_{\Omega}\left|\nabla u_{n}\right|^{p_{1}} \mathrm{~d} x+\left(\frac{1}{p_{2}}-\frac{1}{r}\right) \int_{\Omega}\left|\nabla v_{n}\right|^{p_{2}} \mathrm{~d} x \\
& +C_{7} \int_{\Omega}\left(\left|u_{n}\right|^{\alpha_{1}}+\left|v_{n}\right|^{\alpha_{2}}\right) \mathrm{d} x-\lambda C_{8} \int_{\Omega}\left(\left|u_{n}\right|^{\beta_{1}}+\left|v_{n}\right|^{\beta_{2}}\right) \mathrm{d} x-C_{9} \\
& \geq\left(\frac{1}{p_{1}}-\frac{1}{r}\right) \int_{\Omega}\left|\nabla u_{n}\right|^{p_{1}} \mathrm{~d} x+\left(\frac{1}{p_{2}}-\frac{1}{r}\right) \int_{\Omega}\left|\nabla v_{n}\right|^{p_{2}} \mathrm{~d} x-C_{10},
\end{aligned}
$$

as $n$ large enough, where $C_{i}$ 's are positive constants, which implies that $\left\{\left(u_{n}, v_{n}\right)\right\}$ is bounded.

Claim II. $\left\{\left(u_{n}, v_{n}\right)\right\}$ has a strongly convergent subsequence.

From Claim I, there exists a subsequence, relabel it as $\left\{\left(u_{n}, v_{n}\right)\right\}$, such that

$$
u_{n} \rightarrow u \quad \text { in } W_{0}^{1, p_{1}}(\Omega), \quad v_{n} \rightarrow v \quad \text { in } W_{0}^{1, p_{2}}(\Omega), \quad \text { as } n \rightarrow \infty .
$$

In what follows, we show that

$$
u_{n} \rightarrow u \quad \text { in } W_{0}^{1, p_{1}}(\Omega), \quad v_{n} \rightarrow v \quad \text { in } W_{0}^{1, p_{2}}(\Omega), \quad \text { as } n \rightarrow \infty
$$

In fact, by (3.1) we get

$$
\left\langle\psi^{\prime}\left(u_{n}, v_{n}\right), u_{n}-u\right\rangle \rightarrow 0, \quad \text { as } n \rightarrow \infty
$$

that is,

$$
\left\langle-\Delta_{p_{1}} u_{n}-f_{1}\left(x, u_{n}\right)-\lambda h_{1}\left(x, u_{n}, v_{n}\right), u_{n}-u\right\rangle \rightarrow 0, \quad \text { as } n \rightarrow \infty
$$

From conditions $\left(\mathrm{F}_{1}\right),\left(\mathrm{H}_{1}\right)$, and the compact embedding theorems (see [16]), we have

$$
\left\{\begin{array}{l}
\left|\left\langle f_{1}\left(x, u_{n}\right), u_{n}-u\right\rangle\right| \leq\left\|f_{1}\left(x, u_{n}\right)\right\|_{L^{q_{1}^{\prime}}}\left\|u_{n}-u\right\|_{L^{q_{1}}} \rightarrow 0, \quad \text { as } n \rightarrow \infty, \\
\left|\left\langle h_{1}\left(x, u_{n}, v_{n}\right), u_{n}-u\right\rangle\right| \leq\left\|h_{1}\left(x, u_{n}, v_{n}\right)\right\|_{L^{q^{\prime}}}\left\|u_{n}-u\right\|_{L^{q}} \rightarrow 0, \quad \text { as } n \rightarrow \infty,
\end{array}\right.
$$


where $q_{1}^{\prime}, q^{\prime}$ are the conjugate numbers of $q_{1}, q$, respectively. In combination with (3.5) and (3.6), we obtain

$$
\left\langle-\Delta_{p_{1}} u_{n}, u_{n}-u\right\rangle \rightarrow 0, \quad \text { as } n \rightarrow \infty .
$$

By the property $\left(\mathrm{S}_{+}\right)$of $p$-Laplacian operator (see $\left.[14,17]\right)$, we know that $u_{n} \rightarrow u$ in $W_{0}^{1, p_{1}}(\Omega)$ as $n \rightarrow \infty$. Similarly, we can prove that $v_{n} \rightarrow v$ in $W_{0}^{1, p_{2}}(\Omega)$ as $n \rightarrow \infty$.

\section{Nontrivial solutions}

First, we consider the least energy critical point of the functional $\varphi_{i}$, which is very useful for the multiplicity of nontrivial solutions to system (1.1).

For that purpose, we define

$$
c_{i}^{*}=\inf _{N_{i}} \varphi_{i}, \quad c_{i}^{* *}=\inf _{u \in W_{0}^{1,1, p_{i}}(\Omega) \backslash\{0\}} \max _{t \geq 0} \varphi_{i}(t u) \quad \text { and } \quad c_{i}=\inf _{\gamma \in \Gamma_{i}} \max _{t \in[0,1]} \varphi_{i}(\gamma(t)),
$$

where

$$
\Gamma_{i}=\left\{\gamma \in C\left([0,1], W_{0}^{1, p_{i}}(\Omega)\right) \mid \gamma(0)=0, \varphi_{i}(\gamma(1))<0\right\}, \quad i=1,2 .
$$

Similar to [15], we have the following.

Lemma 4.1 Under conditions $\left(\mathrm{F}_{1}\right)-\left(\mathrm{F}_{4}\right), c_{i}^{*}=c_{i}^{* *}=c_{i}>0$, and $c_{i}$ is a critical value of $\varphi_{i}$.

Proof It is very similar to the proof of Theorem 4.2 on page 73 in [15], so we omit it.

Lemma 4.2 Let $f_{i}$ satisfy conditions $\left(\mathrm{F}_{1}\right)-\left(\mathrm{F}_{4}\right), g_{i}$ be of $C^{2}$ class and satisfy $g_{i}(x, u) \leq C f_{i}(x, u)$ for all $u>0$ with $C>0$ a constant $(i=1,2)$. Denote

$$
G_{i}(x, t)=\int_{0}^{t} g_{i}(x, s) \mathrm{d} s, \quad \psi_{i}(\lambda, u)=\varphi_{i}(u)-\lambda \int_{\Omega} G_{i}(x, u) \mathrm{d} x,
$$

then, on $N_{i}$, we have

$$
\psi_{i}(\lambda, u) \geq(1-\lambda D) \varphi_{i}(u)
$$

for all $\lambda>0$ with $D>0$ a constant.

Proof By the assumptions on $g_{i}$, we have

$$
G_{i}(x, u) \leq C F_{i}(x, u)
$$

for all $u \in \mathbb{R}^{+}$, then

$$
\begin{aligned}
\psi_{i}(\lambda, u) & \geq \varphi_{i}(u)-\lambda C \int_{\Omega} F_{i}(x, u) \mathrm{d} x \\
& =\frac{1}{p_{i}} \int_{\Omega}|\nabla u|^{p_{i}} \mathrm{~d} x-(1+\lambda C) \int_{\Omega} F_{i}(x, u) \mathrm{d} x .
\end{aligned}
$$


Let $D=\frac{p_{i} C}{\alpha_{i}-p_{i}}$, then

$$
\frac{p_{i}(C+D)}{D} \int_{\Omega} F_{i}(x, u) \mathrm{d} x=\alpha_{i} \int_{\Omega} F_{i}(x, u) \mathrm{d} x \leq \int_{\Omega} u f_{i}(x, u) \mathrm{d} x=\int_{\Omega}|\nabla u|^{p_{i}} \mathrm{~d} x
$$

due to $u \in N_{i}$, which means that

$$
\frac{1}{p_{i}} \int_{\Omega}|\nabla u|^{p_{i}} \mathrm{~d} x-(1+\lambda C) \int_{\Omega} F_{i}(x, u) \mathrm{d} x \geq(1-\lambda D) \int_{\Omega}\left[\frac{1}{p_{i}}|\nabla u|^{p_{i}}-F_{i}(x, u)\right] \mathrm{d} x .
$$

The proof is complete.

Theorem 4.1 Assume that $f_{1}, f_{2}$ satisfy conditions $\left(\mathrm{F}_{1}\right)-\left(\mathrm{F}_{4}\right)$ and that $h_{1}, h_{2}$ satisfy conditions $\left(\mathrm{H}_{1}\right)-\left(\mathrm{H}_{2}\right)$ and the following condition:

$\left(\mathrm{H}_{3}\right)$ for a.a. $x \in \Omega$ and all $u, v \in \mathbb{R}^{+}$,

$$
h_{i}(x, u, 0)=h_{i}(x, 0, v)=0, \quad i=1,2 .
$$

Then, there exists a constant $\Lambda>0$ such that system (1.1) has at least three nonnegative solutions for any $\lambda \in(0, \Lambda)$. Furthermore, if the problems

$$
-\Delta_{p_{i}} u=f_{i}(x, u) \quad \text { in } \Omega \text {, with } u=0 \text { on } \partial \Omega(i=1,2)
$$

have both a unique positive solution, then for any $\lambda \in(0, \Lambda)$, system (1.1) has at least three nonnegative solutions in which there is a nontrivial positive solution.

Proof From Lemma 4.1, there exists $w_{i} \in N_{i}$ such that $\varphi_{i}\left(w_{i}\right)=c_{i}$ and $\varphi_{i}^{\prime}\left(w_{i}\right)=0(i=1,2)$, which implies that $\left(w_{1}, 0\right)$ and $\left(0, w_{2}\right)$ are nonnegative solutions of the following system:

$$
\begin{cases}-\Delta_{p_{1}} u=f_{1}(x, u), & \text { in } \Omega, \\ -\Delta_{p_{2}} v=f_{2}(x, v), & \text { in } \Omega, \\ u=v=0, & \text { on } \partial \Omega .\end{cases}
$$

Combining with condition $\left(\mathrm{H}_{3}\right),\left(w_{1}, 0\right)$ and $\left(0, w_{2}\right)$ are nonnegative solutions of system (1.1) with $\psi\left(w_{1}, 0\right)=\varphi_{1}\left(w_{1}\right)=c_{1}$ and $\psi\left(0, w_{2}\right)=\varphi_{2}\left(w_{2}\right)=c_{2}$.

Now, we prove the existence of the third nonnegative solution.

First, we consider $\psi\left(t w_{1}, s w_{2}\right), \forall t, s \geq 1$, as follows:

$$
\begin{aligned}
\psi\left(t w_{1}, s w_{2}\right)= & \frac{1}{p_{1}} \int_{\Omega}\left|\nabla\left(t w_{1}\right)\right|^{p_{1}} \mathrm{~d} x+\frac{1}{p_{2}} \int_{\Omega}\left|\nabla\left(s w_{2}\right)\right|^{p_{2}} \mathrm{~d} x-\int_{\Omega} F_{1}\left(x, t w_{1}\right) \mathrm{d} x \\
& -\int_{\Omega} F_{2}\left(x, s w_{2}\right) \mathrm{d} x-\lambda \int_{\Omega} H\left(x, t w_{1}, s w_{2}\right) \mathrm{d} x \\
= & \frac{1}{p_{1}} \int_{\Omega}\left|\nabla\left(t w_{1}\right)\right|^{p_{1}} \mathrm{~d} x+\frac{1}{p_{2}} \int_{\Omega}\left|\nabla\left(s w_{2}\right)\right|^{p_{2}} \mathrm{~d} x \\
& -\int_{\Omega} F_{1}\left(x, t w_{1}\right) \mathrm{d} x-\int_{\Omega} F_{2}\left(x, s w_{2}\right) \mathrm{d} x
\end{aligned}
$$




$$
\begin{aligned}
& -\lambda \int_{\Omega} \int_{0}^{1}\left[t w_{1} h_{1}\left(x, \tau t w_{1}, \tau s w_{2}\right)+s w_{2} h_{2}\left(x, \tau t w_{1}, \tau s w_{2}\right)\right] \mathrm{d} \tau \mathrm{d} x \\
\leq & \frac{1}{p_{1}} \int_{\Omega}\left|\nabla\left(t w_{1}\right)\right|^{p_{1}} \mathrm{~d} x+\frac{1}{p_{2}} \int_{\Omega}\left|\nabla\left(s w_{2}\right)\right|^{p_{2}} \mathrm{~d} x \\
& -C_{11}\left(\int_{\Omega}\left|t w_{1}\right|^{\alpha_{1}} \mathrm{~d} x+\int_{\Omega}\left|s w_{2}\right|^{\alpha_{2}} \mathrm{~d} x\right) \\
\leq & C_{12}(t+s)^{\max \left\{p_{1}, p_{2}\right\}}-C_{13}(t+s)^{\min \left\{\alpha_{1}, \alpha_{2}\right\}}+C_{14},
\end{aligned}
$$

where $C_{i}$ 's are positive constants, which implies that there exists an $M>0$ such that

$$
\psi\left(t w_{1}, s w_{2}\right)<0, \quad \text { as } t+s \geq M
$$

Let $e_{i}=M w_{i}(i=1,2)$, and

$$
Q=\left\{t e_{1} \mid t \in[0,1]\right\} \times\left\{s e_{2} \mid s \in[0,1]\right\} \quad \text { and } S=N_{1} \times N_{2}
$$

then $\varphi_{1}\left(e_{1}\right)=\psi\left(M w_{1}, 0\right)<0$ and $\varphi_{2}\left(e_{2}\right)=\psi\left(0, M w_{2}\right)<0$, thus $\partial Q$ links $S$ by Theorem 2.2 .

Second, we claim that $\inf _{S} \psi(u, v)>\sup _{\partial Q} \psi(u, v)$ as $\lambda$ is small enough. In fact, by Lemma 4.1,

$$
c_{i}=c_{i}^{* *}=\inf _{u \in W_{0}^{1, p_{i}}(\Omega) \backslash\{0\}} \max _{t \geq 0} \varphi_{i}(t u) \geq \sup _{t \in[0,1]} \varphi_{i}\left(t e_{i}\right),
$$

which, in connection with (4.2), implies that

$$
\sup _{(u, v) \in \partial Q} \psi(u, v) \leq \max \left\{c_{1}, c_{2}\right\}
$$

for all $\lambda>0$.

On the other hand, combining with Lemma 4.2, for any $(u, v) \in S$ we have

$$
\begin{aligned}
\psi(u, v) & =\varphi_{1}(u)+\varphi_{2}(v)-\lambda \int_{\Omega} H(x, u, v) \mathrm{d} x \\
& \geq\left(1-\lambda D_{1}\right) \varphi_{1}(u)+\left(1-\lambda D_{2}\right) \varphi_{2}(v)-\lambda D_{3},
\end{aligned}
$$

here $D_{i}$ 's are proper positive constants. Now let $\Lambda=\min \left\{D_{1}^{-1}, D_{2}^{-1}, \frac{c_{1}+c_{2}-\max \left\{c_{1}, c_{2}\right\}}{c_{1} D_{1}+c_{2} D_{2}+D_{3}}\right\}$, then for all $\lambda \in(0, \Lambda)$,

$$
\inf _{(u, v) \in S} \psi(u, v) \geq\left(1-\lambda D_{1}\right) \varphi_{1}(u)+\left(1-\lambda D_{2}\right) \varphi_{2}(v)-\lambda D_{3}>\max \left\{c_{1}, c_{2}\right\}
$$

Hence, $\inf _{S} \psi(u, v)>\sup _{\partial Q} \psi(u, v)$ is inferred from (4.3) and (4.4).

Finally, we define

$$
c=\inf _{\gamma \in \Gamma} \sup _{Q} \psi(\gamma(u, v)), \quad \text { here } \Gamma=\left\{\gamma \in C\left(Q, W_{0}^{1, p_{1}}(\Omega) \times W_{0}^{1, p_{2}}(\Omega)\right)|\gamma|_{\partial Q}=\left.\mathrm{id}\right|_{\partial Q}\right\}
$$

In combination with Theorem 3.1 and the classical minimax principle (see $[15,18]), c \geq$ $\inf _{S} \psi>\max \left\{c_{1}, c_{2}\right\}$ is the third critical value of $\psi$. 
Therefore, system (1.1) has at least three nonnegative solutions for $\lambda \in(0, \Lambda)$.

In addition, if the equations

$$
\begin{cases}-\Delta_{p_{i}} u=f_{i}(x, u), & \text { in } \Omega, \\ u=0, & \text { on } \partial \Omega\end{cases}
$$

have both a unique positive solution $(i=1,2)$, the third nonnegative solution of system (1.1) is actually positive. In fact, if one component of the third solution equals zero, its functional value equals $c_{1}$ or $c_{2}$, which is a contradiction.

\section{Competing interests}

The authors declare that they have no competing interests.

\section{Authors' contributions}

The authors read and approved the final manuscript.

\section{Acknowledgements}

This work is partly supported by NNSF $(11101404,11201204,11361053)$ of China and the State Scholarship Fund (201308620021) of China Scholarship Council.

\section{Received: 5 October 2013 Accepted: 16 January 2014 Published: 07 Feb 2014}

\section{References}

1. Conti, M, Terracini, S, Verzini, G: Nehari's problem and competing species systems. Ann. Inst. Henri Poincaré, Anal. Non Linéaire 19, 871-888 (2002)

2. Costa, DG, Magalhaes, CA: A variational approach to subquadratic perturbations of elliptic systems. J. Differ. Equ. 111, 103-122 (1994)

3. Hai, DD, Shivaji, R: An existence result for a class of $p$-Laplacian systems. Nonlinear Anal. 56, 1007-1010 (2004)

4. Hai, DD, Wang, H: Nontrivial solutions for $p$-Laplacian systems. J. Math. Anal. Appl. 330, 186-194 (2007)

5. Zhang, J, Zhang, Z: Existence results for some nonlinear elliptic systems. Nonlinear Anal. 71, 2840-2846 (2009)

6. Cheng, X, Ma, S: Existence of three nontrivial solutions for elliptic systems with critical exponents and weights. Nonlinear Anal. 69, 3537-3548 (2008)

7. Cheng, X, Zhong, C: Existence of three nontrivial solutions for an elliptic system. J. Math. Anal. Appl. 327, 1420-1430 (2007)

8. Motreanu, D, Zhang, Z: Constant sign and sign changing solutions for systems of quasilinear elliptic equations. Set-Valued Var. Anal. 19, 255-269 (2011)

9. Shen, Y, Zhang, J: Multiplicity of positive solutions for a semilinear $p$-Laplacian system with Sobolev critical exponent. Nonlinear Anal. 74, 1019-1030 (2011)

10. Zhang, Z, Cheng, X: Existence of positive solutions for a semilinear elliptic system. Topol. Methods Nonlinear Anal. 37 103-116 (2011)

11. Zhao, P, Wang, X: The existence of positive solutions of elliptic system by a linking theorem on product space. Nonlinear Anal. 56, 227-240 (2004)

12. Zhao, P, Zhou, W, Zhong, C: The existence of three nontrival solutions of a class of elliptic systems. Nonlinear Anal. 49 , 431-443 (2002)

13. Ambrosetti, A, Rabinowitz, PH: Dual variational methods in critical point theory and applications. J. Funct. Anal. 14, 349-381 (1973)

14. Dinca, G, Jebelean, P, Mawhin, J: A result of Ambrosetti-Rabinowitz type for $p$-Laplacian. In: Corduneanu, C (ed.) Qualitative Problems for Differential Equations and Control Theory. World Scientific, Singapore (1995)

15. Willem, M: Minimax Theorems. Birkhäuser, Basel (1996)

16. Adams, RA: Sobolev Spaces. Academic Press, New York (1975)

17. Dinca, G, Jebelean, $\mathrm{P}, \mathrm{Mawhin}, \mathrm{J}$ : Variational and topological methods for Dirichlet problems with $p$-Laplacian. Port. Math. 58, 339-378 (2001)

18. Mawhin, J, Willem, M: Critical Point Theory and Hamiltonian Systems. Springer, Berlin (1989)

10.1186/1687-2770-2014-34

Cite this article as: Cheng and Yang: Nontrivial solutions for a quasilinear elliptic system. Boundary Value Problems 2014, 2014:34 